\title{
Model-based Delineation of Non-Uniformly Sampled ECG Signals
}

\author{
Thomas Niederhauser ${ }^{1}$, Andreas Haeberlin ${ }^{2,3}$, Barbara Jesacher ${ }^{1}$, Andreas Fischer ${ }^{4,5}$, Hildegard \\ Tanner $^{3}$ \\ ${ }^{1}$ Institute for Human Centered Engineering, Bern University of Applied Sciences, Biel, Switzerland \\ ${ }^{2}$ ARTORG Center for Biomedical Engineering Research, University of Bern, Bern, Switzerland \\ ${ }^{3}$ Department of Cardiology, Inselspital, Bern University Hospital, Bern, Switzerland \\ ${ }^{4}$ DIVA research group, University of Fribourg, Fribourg, Switzerland \\ ${ }^{5}$ iCoSys Institute, University of Applied Sciences HES-SO, Fribourg, Switzerland
}

\begin{abstract}
Level-crossing Electrocardiography (ECG) signal acquisition provides many advantages with respect to power consumption and memory requirements suitable for longterm recording. In this paper, we investigated the delineation accuracy of non-uniform ECG signals arising from level-crossing sampling based on a hidden Markov model (HMM) and compared the results with conventional, uniform sampling. Dedicated signal features were extracted to emphasize the important ECG waves, i.e. the QRScomplex, the $P$ and the T-wave. The HMM-based segmentation applied to annotated ECGs from the QT-database showed comparable results for both sampling methods. However, the delineation error of the R-peak observed in the non-uniform data is smaller than in the uniform data (3.2 vs. $4.0 \mathrm{~ms}$ ) even for a lower average sampling rate $(69$ vs. 250 samples per second). Level-crossing sampling in combination with HMM-based signal segmentation is indicated to be a suitable tool for prolonged ECG recording featuring high delineation accuracy.
\end{abstract}

\section{Introduction}

Atrial fibrillation is the most common heart rhythm disorder (arrhythmia) and is associated with severe outcomes such as stroke. Short and rare episodes of irregular heartbeats, e.g. paroxysmal atrial fibrillation, require long-term ECG recording to establish the correct diagnoses along with the therapeutic strategy.

The automatic detection and segmentation of the QRS complex, $\mathrm{P}$ and $\mathrm{T}$ wave within the ECG is crucial to analyze long-term recordings. However, ECG wave delineation is affected by signal noise and artifacts resulting from muscle activity, cable and electrode motion. Many researchers have proposed filter or model-based approaches such as wavelet transformation [1] or extended
Kalman filtering [2] to improve the delineation accuracy. Unfortunately, the proposed algorithms assume a stringent time relation between the less pronounced $\mathrm{P}$ and $\mathrm{T}$ wave and the pre-detected QRS complex, which makes the delineation of irregular heartbeats cumbersome.

Smart recorders such as wearable ECG patches, functional clothes, or implantable loop recorders have recently attracted much attention. They provide the advantage to be leadless which may reduce signal artifacts. However, such devices need to be highly memory- and power-efficient to fulfill the space requirements at the site of application. In the case of surface recorders, storage memory can be saved by wireless communication within a body area network with the drawback to give up the autonomy of the wearable recorder. Implantable recorders just store predefined events. The online event detection in turn increases the power consumption and reduce the reliability of the longterm recording since the continuous ECG signal cannot be screened offline.

Level-crossing sampling may provide a way out. In contrast to conventional, Nyquist-based (uniform) sampling, a new sample is acquired only in case the input signal undergoes a significant change. This activity-based sampling is rather simple to integrate into low-power electronics [3] and provides high memory efficiency when operating on burst-like signals that are sparse in time, like the ECG as shown in Figure 1. Furthermore, the characteristics of non-uniform samples enables subsequent data compression stages to be very power-efficient [4]. Non-uniform signal acquisition has already been used in applications such as speech, ultrasound, and accelerometer processing as well as to biomedical signals such as the online detection of the QRS complex [5].

In this paper, we focus on the offline processing of nonuniform ECG signals. Due to the pseudo-periodic characteristics of the ECG a model-based delineation based on a Markov chain is feasible. We propose to use a time- 


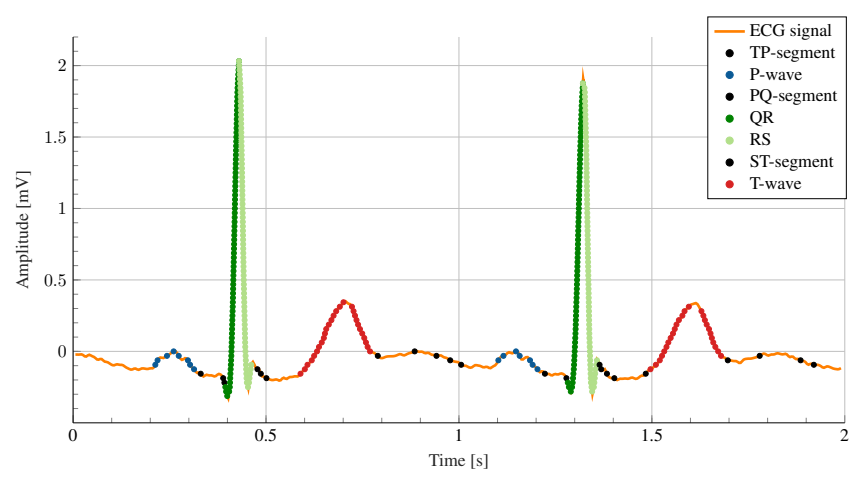

Figure 1. ECG signal and sampling points obtained with level-crossing sampling.

unconstrained hidden Markov model (HMM), that is, a model with hidden states that represent the ECG waves without knowing the wave boundaries a priori. HMMs are widely used in pattern classification such as handwriting recognition or keyword spotting [6] where the exact start and end points of letters are neither known. The HMM approach has been successfully applied to ECG rhythm classification based on uniform samples [7], as well.

\section{Methods}

\subsection{Hidden Markov Model}

The HMM is a stochastic state machine based on a stationary and causal Markov chain and can be used to generate an observation sequence $\boldsymbol{O}=\left[o_{k}\right]_{1 \times K}$. The size of the observation vector $o_{k}$ is related to the number of distinct observation symbols representing the signal. A HMM can be characterized by the subsequent parameter set:

$$
\boldsymbol{\Lambda}=(\boldsymbol{A}, \boldsymbol{B}, \boldsymbol{\pi}),
$$

where $\boldsymbol{A}=\left[a_{i j}=P\left(s_{j_{t}} \mid s_{i_{t-1}}\right)\right]_{N \times N}$ is the matrix of state-transition probabilities, $\boldsymbol{B}=\left[b_{j k}=P\left(o_{k} \mid s_{j}\right)\right]_{N \times K}$ the matrix of observation probabilities, and $\pi=P\left(s_{i_{t=1}}\right)$ are the initial state probabilities, with $N$ and $K$ equals the number of states and observations, respectively.

The HMM consists of hidden states $S=\left[s_{i}\right]_{1 \times N}$. To model the ECG signal, these states are interconnected such that each state can be reached from any previous state, named the Bakis model as depicted in Figure 2.

\subsection{Emulator for Level-Crossing Sampling}

The idea is to use annotated ECG signals derived from a public-domain database. However, these ECGs are uniformly sampled and need to be transformed to nonuniform samples by a software-based emulation of the level-crossing scheme. The emulator was implemented in MATLAB (Mathworks, USA) and works as follows: First, the powerline interference is suppressed with a Notch-filter and a wavelet decomposition is applied to get rid of high frequency noise. Second, the filtered signal commonly sampled with $250 \mathrm{~Hz}$ is upsampled to $32 \mathrm{kHz}$ to receive an analog-like signal. Third, the level-crossing sampling is applied with voltage resolutions $d V=1 / 2^{N} \mathrm{mV}$ where $N=\{3.5,4,4.5,5,5.5,6\}$ bits/mV. A signal sample is acquired only when the absolute voltage difference between the analog value and the previous non-uniform sample exceeds $d V$.

\subsection{Feature Set}

The data-set includes 23 ECG signals extracted from the QT-database (physionet.org), each providing the first 120 heartbeats fully annotated. Apart from potential STsegment changes, we have selected physiological heartbeats only. 33 features were extracted from non- and uniformly sampled ECG signals. The feature vector $F$ for the non-uniform data was defined as

$$
\vec{F}=\left[\begin{array}{c}
d S_{i} \\
d d S_{i} \\
s p_{i} \\
\sum^{j} d S_{i} \\
\sum^{j} d d S_{i} \\
\sum^{j} s p_{i}
\end{array}\right]=\left[\begin{array}{c}
S_{i+1}-S_{i} \\
d S_{i+1}-d S_{i} \\
\pm d V \\
\sum_{i}^{i+10 j} d S_{i} \\
\sum_{i}^{i+10 j} d d S_{i} \\
\sum_{i}^{i+10 j} s p_{i}
\end{array}\right], \quad \forall j \in[1,10],
$$

where $S$ is the number of cycles, counted on the basis of $32 \mathrm{kHz}$, between two subsequent level-crossings that show

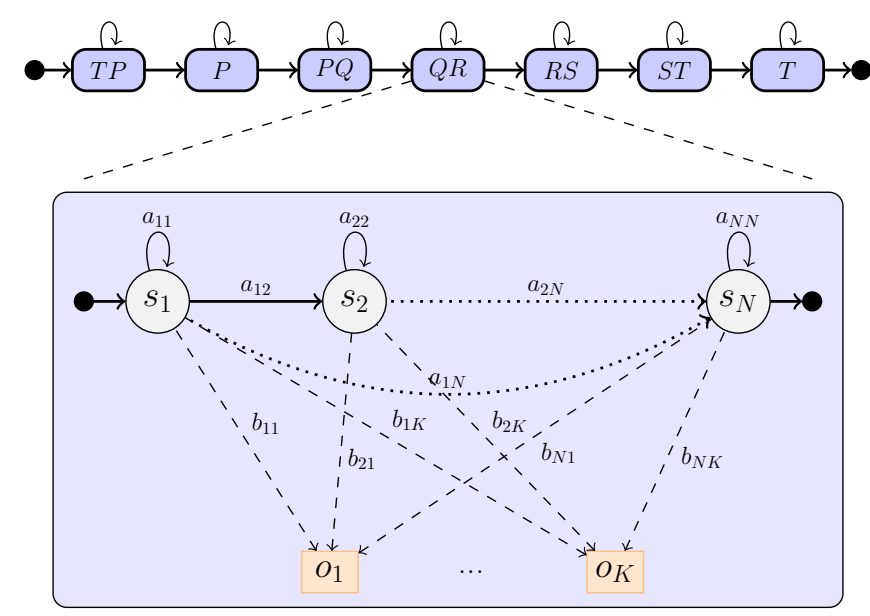

Figure 2. Overview of the model-based ECG segmentation using one HMM for each ECG wave (top) and the Bakis model with states $s_{i}$, output $o_{k}$ and corresponding probabilities exemplary shown for the QR wave. 
either a positive or negative voltage step (equals $s p$ ). $M$ equals the total number of samples within one ECG. For the uniform data, a similar feature vector was used replacing the cycles $S$ by the voltage amplitude $V$.

\subsection{Implementation and Training}

We used the toolkit (HTK) which is a portable library for building and manipulating HMM. Although HTK is dedicated to speech recognition, it can be used to segment ECG signals, as well. Each ECG wave (P wave, PQ segment, $\mathrm{QR}$ wave, RS wave, ST segment, $\mathrm{T}$ wave and TP segment) correspond to a word in speech. The ECG waves are linked with the left-right HMM topology shown in Figure 2 representing a physiological heartbeat. The concatenation of multiple heartbeats reproduce the pseudo-periodic ECG signal as a whole.

The HMM parameter set was estimated with the BaumWelch method [8] (expectation-maximization method). Each of the 7 HMMs (see Figure 2) was trained on its respective ECG wave using the first 60 annotated heartbeats of each ECG record individually. The number of states representing each ECG wave was dependent on the number of samples or the duration of the wave in case of nonuniform and uniform data, respectively. It should be noted that we artificially increased the number of data points to a minimum number of 250 per second such that the training was adequate for ECG waves featuring a few non-uniform samples only. Observation sequences were modeled as a mixture of three Gaussian functions.

\subsection{Testing and Performance Evaluation}

The second half of each ECG record containing 60 annotated heartbeats was used as test data. With the Viterbi algorithm [8], we got the most likely sequence of states given the observation sequence, i.e. the non-uniform or uniform data. This sequence of states is then transformed into subsequent ECG waves and subsequent heartbeats.

We evaluated the on- and offsets of all ECG waves with respect to the reference annotations. The R-peak detected as the transmission between $\mathrm{QR}$ and RS wave was compared to the amplitude peak searched within an interval of $\pm 50 \mathrm{~ms}$ around the reference annotation. Statistical comparisons were done with the Wilcoxon signed rank test for paired probes using a significance level of $5 \%$ and Bonferroni correction for multiple testing.

\section{Results}

Figure 3 shows the evaluation of the trained HMM applied to the 23 ECG signals containing 60 testing heartbeats each. The delineation error (median \pm iqr) between the R-peak, onset and offset of detected ECG waves and the reference annotations is illustrated for both, the nonuniform and uniform samples.

Non-uniformly sampled ECG signals exhibit a total delineation error that is slightly affected by the average sampling rate. The error decreases from $6.1 \pm 6.2 \mathrm{~ms}$ to $5.7 \pm 5.5 \mathrm{~ms}$ with increasing sampling rate and accordingly increasing sampling resolution, but is not different to the total error of $6.1 \pm 3.8 \mathrm{~ms}$ achieved with uniform sampling (all $p>0.03$ ).

Having a closer look on the individual wave segmentation, we observe that the QRS complex comprising QR and RS waves is more accurately delineated than the two other ECG waves, in particular the $\mathrm{T}$ wave. The R-peak localization shows an error of $3.2 \pm 0.8 \mathrm{~ms}$ for the lowest sampling resolution $N=3.5 \mathrm{bits} / \mathrm{mV}$ that decreases to $1.1 \pm 0.7 \mathrm{~ms}$ for $N=6 \mathrm{bits} / \mathrm{mV}$. Regardless of the non-uniform sampling resolution, the localization is more accurate as compared to the uniform ECG samples having an error of $4.0 \pm 0.9 \mathrm{~ms}$ (all $p<6 \cdot 10^{-5}$ ). T-offset manifests the highest delineation error of $15.2 \pm 20.5 \mathrm{~ms}$ and $8.4 \pm 12.1 \mathrm{~ms}$ at sampling resolutions of $N=3.5 \mathrm{bits} / \mathrm{mV}$ and $N=6$ bits $/ \mathrm{mV}$, respectively. However, the error observed with uniform ECG samples is in a similar range, $11.7 \pm 14.3 \mathrm{~ms}$.

The distribution of the sampling points among the ECG signal changes with level-crossing sampling. The average number of sampling points observed within the nonuniform QRS complex is 2 to 12 times higher - depending on the sampling resolution - than for the uniformly sampled counterparts containing 26 sampling points on average. In contrast, the non-uniform idle segments (PQ, ST, TP segment) contain less sampling points (around 2 to 85 times less) compared to uniform samples.

\section{Discussion \& Conclusion}

We have successfully applied a dedicated HMM topology using the HTK toolbox for segmenting non-uniformly sampled ECG signals into its elementary waves. The Rpeak, ECG wave onset and offsets have been evaluated and compared with reference annotations.

With respect to the segmentation of the entire heartbeat, we note that the delineation error resulting from nonuniform samples was comparable to that of uniform samples. However, the average sampling rate with resolution $N=3.5$ bits $/ \mathrm{mV}$ drops more than $70 \%$ from 250 to 69 samples per second. Level-crossing sampling concentrates the acquired values within a heartbeat on signal portions with a steep slope. On- and offset of the QRS complex, the $\mathrm{P}$ and $\mathrm{T}$ wave as well as fast changes within these waves are emphasized. In contrast, the PQ, ST, and TP segment produce much less samples. This behavior supports a more specific training of the HMMs reducing the required samples. The lowest error can be found for the QRS complex 


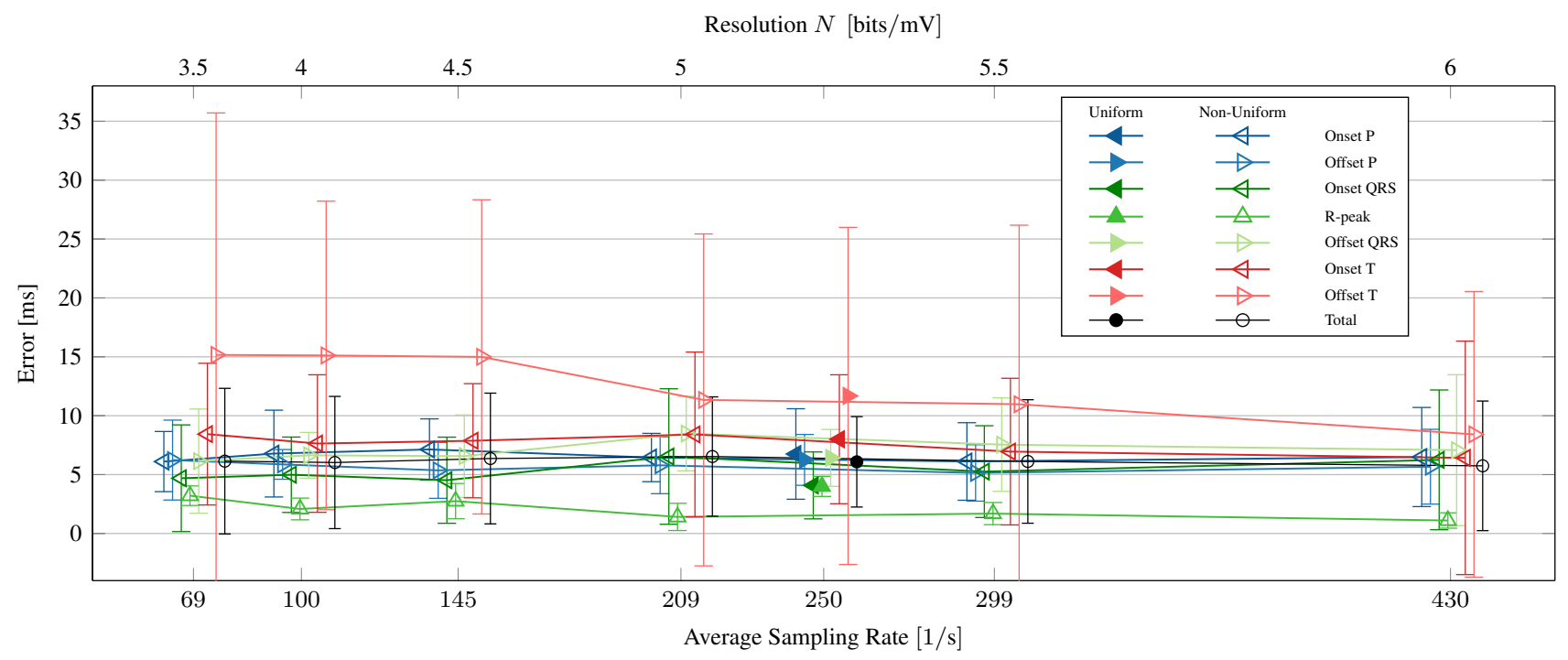

Figure 3. Median error and interquartile range as a function of the average sampling rate (lower x-axis) and corresponding sampling resolution (upper x-axis) of the R-peak, ECG wave onsets and offsets of the non-uniformly sampled signals (non-filled markers). For comparison, the result of the HMM applied to uniform samples (filled markers) is shown.

delineation due to its steep slope resulting in a very high number of sampling points, i.e. a low time interval between the non-uniform samples. In contrast, the largest error was observed for the T-offset. This is most likely associated with the poor reference annotations and is not related to the HMM-based segmentation. The error observed for the uniform samples is in the same range.

In summary, HMM-based delineation of non-uniformly sampled ECG signals shows a high accuracy even for low average sampling rates. Future investigations will consider time-constraint states (e.g. by the duration of ECG waves) to improve the delineation further. We also will apply the HMM based segmentation on ECG signals with atrial fibrillation. This can be achieved by testing various heartbeat models with a different number and order of HMMs (ECG waves) than shown in Figure 2 and choosing the most likely model for a given ECG sequence.

\section{Acknowledgements}

This research project was funded by the Swiss Heart Foundation.

\section{References}

[1] Martinez JP, Almeida R, Olmos S, Rocha AP, Laguna P. A wavelet-based ECG delineator: evaluation on standard databases. IEEE Transactions on Biomedical Engineering April 2004;51(4):570-581. ISSN 0018-9294.

[2] Sayadi O, Shamsollahi MB. A model-based Bayesian framework for ECG beat segmentation. Physiological Measurement March 2009;30(3):335. ISSN 0967-3334.
[3] Marisa T, Niederhauser T, Haeberlin A, Wildhaber RA, Vogel R, Goette J, Jacomet M. Pseudo Asynchronous Level Crossing adc for ecg Signal Acquisition. IEEE Transactions on Biomedical Circuits and Systems 2017;PP(99):112. ISSN 1932-4545.

[4] Marisa T, Niederhauser T, Haeberlin A, Wildhaber RA, Vogel R, Jacomet M, Goette J. Bufferless Compression of Asynchronously Sampled ECG Signals in Cubic Hermitian Vector Space. IEEE Transactions on Biomedical Engineering December 2015;62(12):2878-2887. ISSN 0018-9294.

[5] Zhang X, Lian Y. A 300-mV 220-nW Event-Driven ADC With Real-Time QRS Detection for Wearable ECG Sensors. IEEE Transactions on Biomedical Circuits and Systems December 2014;8(6):834-843. ISSN 1932-4545.

[6] Fischer A, Keller A, Frinken V, Bunke H. Lexicon-free handwritten word spotting using character HMMs. Pattern Recognition Letters May 2012;33(7):934-942. ISSN 0167-8655.

[7] Andreao RV, Dorizzi B, Boudy J. ECG signal analysis through hidden Markov models. IEEE Transactions on Biomedical Engineering August 2006;53(8):1541-1549. ISSN 0018-9294.

[8] Rabiner L. A tutorial on hidden Markov models and selected applications in speech recognition. Proceedings of the IEEE February 1989;77(2):257-286. ISSN 0018-9219.

Address for correspondence:

Thomas Niederhauser

Institute for Human Centered Engineering

Bern University of Applied Sciences

Quellgasse 21, 2501 Biel, Switzerland

thomas.niederhauser@bfh.ch 